\title{
The Use of NanoComputed Tomography to Enhance Musculoskeletal Research
}

\author{
Basma Khoury ${ }^{1}$, Erin M. R. Bigelow ${ }^{1}$, Lauren M. Smith ${ }^{1}$, Robert W. Goulet ${ }^{1}$, Erica Scheller ${ }^{2}$, Nelly \\ Andarawis-Puri ${ }^{3}$, Karl J. Jepsen ${ }^{1}$
}

${ }^{1}$ Department of Orthopaedic Surgery, University of Michigan, Ann Arbor, MI USA

${ }^{2}$ Molecular \& Integrative Physiology, University of Michigan, Ann Arbor, MI USA

${ }^{3}$ Department of Orthopaedics, Mount Sinai School of Medicine, New York, NY USA

MicroComputed Tomography (microCT) has long been used in medical research, primarily to quantify the structural and compositional (density) properties of bone [1-3]. This technology has transformed our ability to quantify changes in bone structure following genetic and environmental perturbations (e.g., age-related bone loss contributing to osteoporosis). Prior to the commercialization of these systems, information about bone structure was determined primarily through histological procedures, which are expensive, labor intensive, and increase the chemical burden. Although histological procedures will remain critical to address cellular information, microCT, in conjunction with Finite Element Analysis, has improved our ability to identify biomechanical mechanisms of disease.

The introduction of nanoComputed Tomography (nanoCT) will also be transformative, not simply for increased resolution, but because the system has enabled us to expand beyond bone and to address scientific questions in a more integrative manner. Our system (nanotom-s; phoenix|x-ray, GE Measurement \& Control; Wunstorf, Germany) has allowed us to expand our musculoskeletal research program in terms of time, scale, and tissue type. First, the nano-focused beam allows us to image bones at much earlier ages when many diseases originate. Pre- and post-natal skeletal structures are poorly attenuating owing to their small size and low mineralization, which makes it very difficult to generate images for quantifying bone morphology using conventional microCT systems. Second, newer imaging platforms have allowed us to image a wider range of bone and joint sizes, from 1 day old mouse femora $(1 \mathrm{~mm})$ to proximal human femurs $(100 \mathrm{~mm})$ (Fig 1). This diversity is particularly important for supporting a translational research programs. Although increased resolution is generally associated with reduced sample volume, our nanoCT system uses motorized detectors and advanced stitching software to generate larger volumes without loss of resolution. Third, nanoCT, in conjunction with select contrast enhancements, have allowed us to expand well beyond bone and to also image muscle, tendon, ligament, cartilage, adipose tissue, blood vessels, and nerves (Figs 2, 3). Further, the high resolving capability of the nanoCT has allowed us to quantify the size and shape of individual adipocytes and then to compare the size distribution across scientific cohorts.

Thus, we are using nanoCT as a way to expand beyond bone, to understand how multiple systems respond to a perturbation while in their anatomical orientation, to identify mechanisms of disease that arise outside the region of interest, and to promote interactions among scientists. One of the challenges associated with nanoCT images involves managing and analyzing large data files (transfer, reconstruction, storage). Further, our ability to image multiple tissue-types has exceeded the ability of software to anatomically dissect the various tissue types in an automated (or semi-automated) manner.

\section{References}

[1] Feldkamp et al, J Bone Miner Res 1989, 4:3-11. 
[2] Kuhn et al, J Orthop Res 1989, 7:876-884.

[3] Kuhn et al, J Orthop Res 1990, 8:833-842.

[4] Scheller et al, Methods Enzymol 2014, 537:123-139.

[5] Pauwels et al, J Microsc 2013, 250:21-31.

[6] This work was supported by a Shared Instrumentation Grant from the NIH (RR026336)

Fig 1. The nanoCT allows a large variety of specimens of different sizes to be scanned. (A) A single embryonic mouse skeleton. Scanning parameters of $8.5 \mu \mathrm{m}$ voxel size, $90 \mathrm{kV}, 390 \mu \mathrm{A}, 750 \mathrm{~ms}$ exposure time. (B) An adult human proximal femur. Scanning parameters of $27 \mu \mathrm{m}$ voxel size, $110 \mathrm{kV}, 200 \mu \mathrm{A}$, $1000 \mathrm{~ms}$ exposure time.
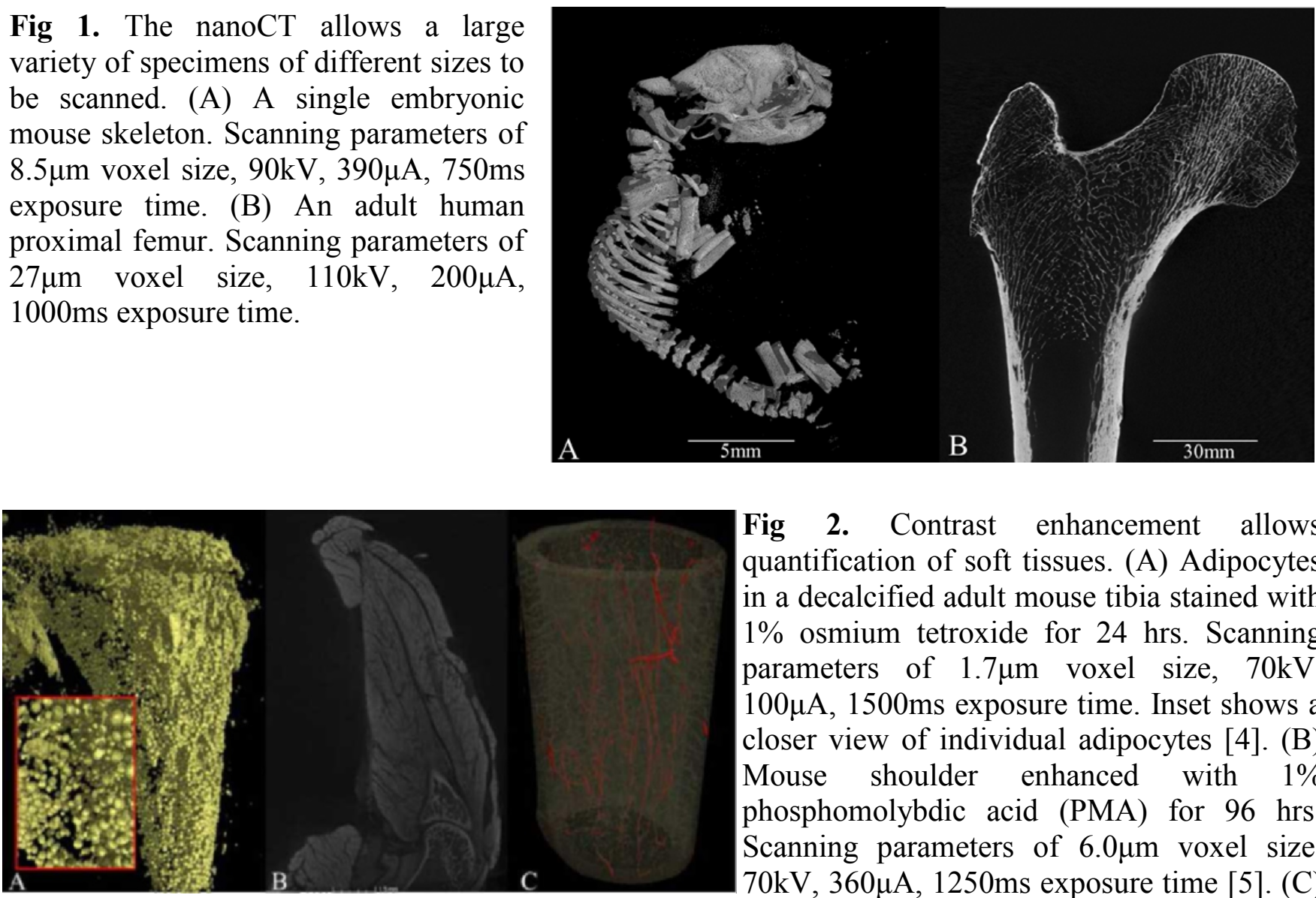

Fig 2. Contrast enhancement allows quantification of soft tissues. (A) Adipocytes in a decalcified adult mouse tibia stained with $1 \%$ osmium tetroxide for $24 \mathrm{hrs}$. Scanning parameters of $1.7 \mu \mathrm{m}$ voxel size, $70 \mathrm{kV}$, $100 \mu \mathrm{A}, 1500 \mathrm{~ms}$ exposure time. Inset shows a closer view of individual adipocytes [4]. (B) Mouse shoulder enhanced with $1 \%$ phosphomolybdic acid (PMA) for $96 \mathrm{hrs}$. Scanning parameters of $6.0 \mu \mathrm{m}$ voxel size, $70 \mathrm{kV}, 360 \mu \mathrm{A}, 1250 \mathrm{~ms}$ exposure time [5]. (C) Mouse femur perfused with $10 \%$ barium sulfate. The blood vessels are highlighted in red against the semi-transparent cortical bone. Scanning parameters of $1.55 \mu \mathrm{m}$ voxel size, $70 \mathrm{kV}, 375 \mu \mathrm{A}, 2000 \mathrm{~ms}$ exposure time.
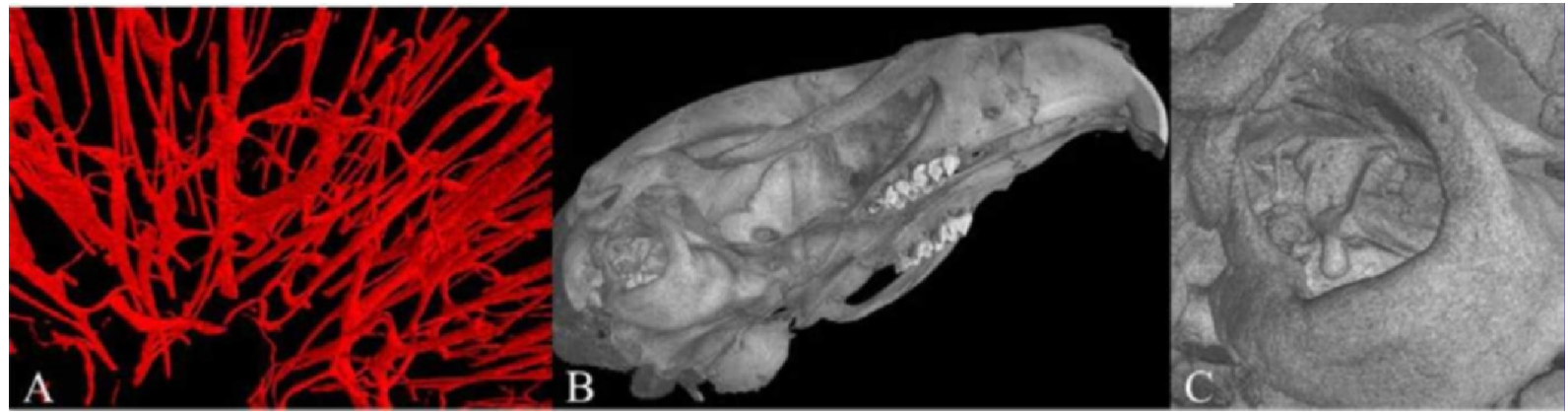

Fig 3. Additional nanoCT applications. (A) Porosity from a small segment of the human tibia. Scanning parameters of $5.0 \mu \mathrm{i}$ voxel size, $120 \mathrm{kV}, 140 \mu \mathrm{A} 1000 \mathrm{~ms}$ exposure time. (B) Rat skull scanned at $6.0 \mu \mathrm{m}$ voxel size, $80 \mathrm{kV}, 310 \mu \mathrm{A} 1000 \mathrm{~ms}$ exposure time. (C) A closer view of the same reconstructed rat skull clearly shows the stapes, malleus, and incus of the rat. 